\title{
Is prefrontal cortex found only in mammals?
}

Over the last twenty years, it has become clear that the organizations of the telencephala of birds and mammals are more similar than was once realized. Recently, Divac and Mogenson have proposed that pigeons possess a telencephalic region that corresponds both anatomically and functionally to mammalian prefrontal cortex. Although this idea requires further experimental scrutiny, it raises the possibility that prefrontal cortex may not be unique to mammals.

Our understanding of the evolution of the vertebrate telencephalon has advanced considerably since the early part of this century. The older notion, which we now know to be incorrect, was that the basal ganglia had hypertrophied enormously in the avian lineage and had come to constitute almost the entire extent of the telencephalon ${ }^{1}$. Since at that time the basal ganglia were thought to be responsible for the control of instinctive species-specific behaviors, their domination of the avian telencephalon was thought to render birds largely incapable of flexible, adaptive behavior.

During the late 1960 s experimental neuroanatomical studies revealed that the basal ganglia do not in fact dominate the telencephalon in birds ${ }^{3-5}$ but instead make up approximately the same proportion of the telencephalon as found in mammals ${ }^{2}$. This discovery quickly led to questions regarding the nature of the vast expanse of the avian telencephalon that overlies and surrounds the basal ganglia (see Fig. 1). During the last twenty years, many studies (most notably those by $\mathbf{H}$. J. Karten $^{6}$ and W. Hodos ${ }^{7}$ and their various collaborators) have shown that this portion of the telencephalon (which consists of two major morphological subdivisions, the Wulst and the dorsal ventricular ridge), has connections and subserves functions characteristic of mammalian neocortex. For example, distinct subfields of the avian telencephalon receive input from sensory relay nuclei of the thalamus, and other distinct subfields are the source of major descending projections to brainstem motor and premotor cell groups (see Fig. 1). Many functional studies have shown that the Wulst and dorsal ventricular ridge (DVR) play a major and indispensable role in higher order sensory processing, and in learning and initiating complex behaviors (for example, song learning and production) ${ }^{f-9}$. However, the Wuist and DVR

*Although the Wulst, particularly in owls, does show a semblance of laminar organization, this is distinctly different from the laminar organization of the mammalian neocortex. do not show the type of laminar organization exhibited by mammalian neocortex but instead are largely organized as a field of contiguous cell groups* .

Against this backdrop, recent work by Divac and his co-workers ${ }^{10,11}$ is of interest because it implies that the similarities between the avian and mammalian telencephala are even greater than currently recognized. Based on histochemical, biochemical and behavioral studies, they report that a region resembling mammalian prefrontal cortex is present in the pigeon telencephalon. To reach this conclusion, they have used a line of reasoning that has become standard among evolutionary neuroscientists interested in comparing the brain structures of living species. Having previously investigated the anatomical, histochemical

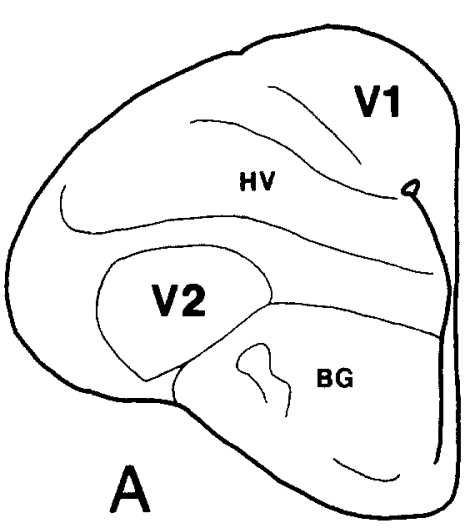

and biochemical organization and function of the prefrontal cortex in mammals $^{12,13}$, Divac and his coworkers recognized several invariant characteristics of prefrontal cortex: (1) the entirety of prefrontal cortex (and no other cortical region) receives input from the dorsomedial thalamic nucleus (as has been traditionally recognized) ${ }^{14}$; (2) the prefrontal cortex receives a prominent dopaminergic input from the $A 9$ and A10 cell groups of the tegmentum; and (3) the prefrontal cortex is the only cortical area that is indispensable for the performance of tasks that involve a delay between the occurrence of a discriminative stimulus and the appropriate response, such as delayed alternation.

Although prefrontal cortex derives its name from its position at the rostral pole of the cerebral hemispheres in primates (anterior to the motor and premotor cortices), Divac and his coworkers found that prefrontal cortex varies considerably in its location and extent among mammalian species. Thus, they concluded that topographic

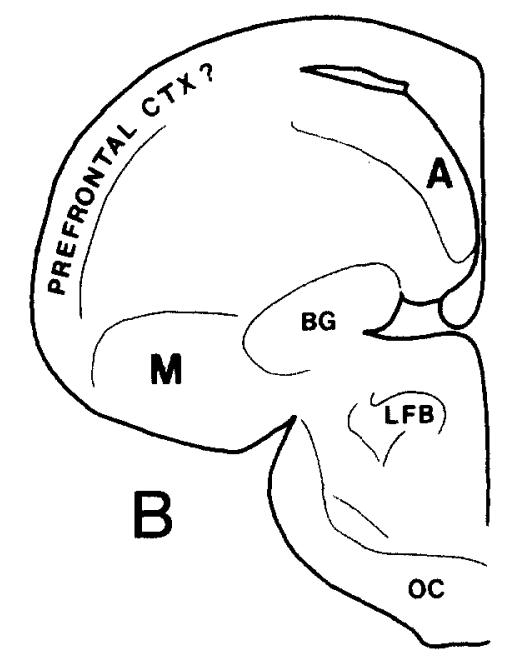

Fig. 1. The avian basal ganglia $(B G)$ are surrounded by a large expanse of telencephalic tissue that shows many of the connections and functional properties of mammalian neocortex. For example, at more rostral telencephalic levels (A), two visual thalamorecipient areas are present. The region designated VI (termed the visual Wulst) receives input from a retinorecipient thalamic nucleus that resembles the mammalian dorsal lateral geniculate in its connections and topographic location. Thus, VI is comparable to mammalian striate cortex. The region designated V2 (termed the ectostriatum) receives input from nucleus rotundus, which receives a massive visual input from the tectum. In these respects, nucleus rotundus resembles the lateral posterior nucleus (or inferior pulvinar) of mammals, and V2, therefore, resembles extrastriate visual cortex of mammals. More caudally (B), a thalamorecipient auditory area (that receives input from a thalamic cell group that itself receives input from the inferior colliculus-homologue) is present, as is an area that gives rise to major descending projections to motor and premotor cell groups of the midbrain and hindbrain. The auditory area (termed Field $L$ and designated $A$ in this figure) is thus comparable to primary auditory cortex of mammals and the motor area (termed the archistriatum and designated $M$ in this figure) shows some resemblance to motor cortex. Divac and Mogenson suggest a further resemblance of the avian telencephalon to that of mammals by proposing that posterodorsolateral portions $(P D L)$ of the telencephalon are comparable to prefrontal cortex, based on criteria discussed in the text. Other abbreviations: $L F B$, lateral forebrain bundle; $O C$, optic chiasm. 
location was a poor criterion for identifying prefrontal cortex. Following studies using the three criteria above to demonstrate the presence and location of prefrontal cortex in a wide variety of mammalian species, Divac became interested in the evolutionary antiquity of the prefrontal cortex. He and his coworkers therefore decided to look for a prefrontal cortex in pigeons (which are used extensively for neuroanatomical studies of the avian nervous system). Since the identity of the avian equivalent of the mammalian dorsomedial thalamic nucleus is unknown, the first criterion could not be used. Turning to the second criterion, Divac and Mogenson used catecholamine histofluorescence and found three regions (Figs 1 and 2) in the Wulst or DVR that were rich in dopaminergic (DA) fibers: (1) a thin, restricted rostral portion of the hyperstriatum ventrale of the DVR; (2) a dorsal portion of a caudolateral motor output area of the DVR termed the archistriatum; and (3) a posterodorsolateral telencephalic field (PDL) within the DVR with a large rostrocaudal extent. Since the DA fiber-containing portion of the hyperstriatum ventrale was not extensive and since Divac and Mogenson considered the DA fibers in this region to represent fibers of passage en route to the PDL, they concluded that this portion of the hyperstriatum ventrale was an unlikely candidate for avian prefrontal cortex. Since the archistriatum is a motor output field with extensive descending projections to the hindbrain ${ }^{4}$ (which is not true of prefrontal cortex), the archistriatum was also thought to be unlikely as an equivalent of prefrontal cortex. This left PDL as a possible candidate. In a separate set of biochemical studies ${ }^{10}$, Divac and his coworkers confirmed that PDL was rich in dopamine, thereby indicating that the catecholaminergic fibers observed in PDL in the histofluorescence studies did reflect the presence of a prominent dopaminergic (as opposed to a noradrenergic) input. Although Divac and Mogenson did not investigate the source of the dopaminergic input to PDL, another study has shown that the input does appear to arise from the dopaminergic cell groups of the avian tegmentum that correspond to the mammalian A9 and A10 cell groups ${ }^{15}$.

Turning to the third criterion, Divac and his co-workers examined the functional similarity of PDL to prefrontal cortex by determining the effects

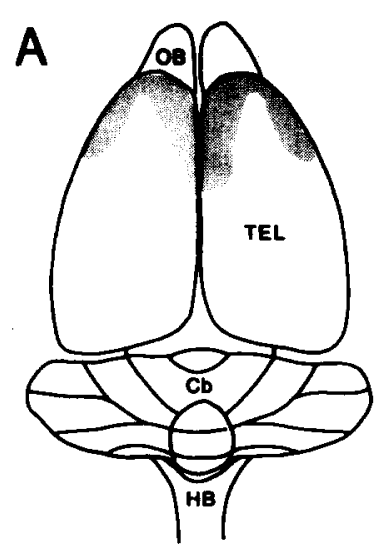

C

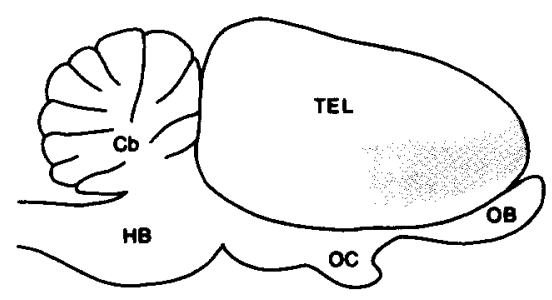

D

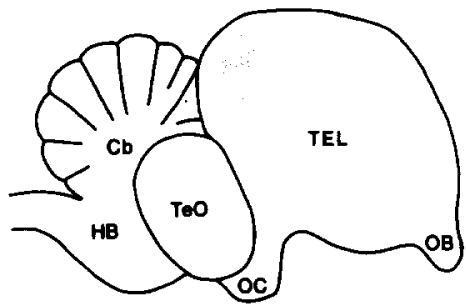

Fig. 2. Schematic illustrations showing the location of prefrontal cortex in a dorsal view (A) and a side view (C) of a rat brain as compared to the location of the putative prefrontal cortex of birds as seen in a dorsal view (B) and side view (D) of a pigeon brain. The shaded areas illustrate the location of prefrontal cortex. The bars in $(B)$ indicate the levels illustrated in Fig. $1(A)$ and $1(B)$. Abbreviations: $O B$, olfactory bulb; $T E L$, telencephalon; $C b$, cerebellum; $H B$, hindbrain; $T e O$, optic tectum; $O C$, optic chiasm.

of PDL lesions on the performance of pigeons in a delayed alternation task ${ }^{11}$. In this task the correct response alternated between two possible responses from trial to trial, with no overt cue present to signal the correct response. The pigeon was required to alternate its responses accordingly to obtain a grain reward. To respond correctly, a bird had to remember the previous correct response and then perform the alternate response. Extensive damage to PDL was found to impair pigeons in their performance of this task but not in the performance of a visual discrimination task.

Based on these similarities, Divac and Mogenson concluded that the PDL was the avian prefrontal cortex. However, they noted that the data were insufficient to determine whether avian PDL and mammalian prefrontal cortex are homologous - that is, that they had been inherited from a reptilian common ancestor, which also possessed a prefrontal cortex - or whether PDL and prefrontal cortex serve analogous functions but have evolved independently. Since prefrontal cortex has been thought to be a uniquely mammalian structure either possibility is of con- siderable interest. If the former is true, Divac's work raises the possibility that prefrontal cortex may be a characteristic of the brain of living reptiles. However, it would also be of interest if PDL and prefrontal cortex subserve similar functions and have independently evolved similar anatomical organization to do so, for this would suggest that specific anatomical features, such as prominent dopaminergic input from the tegmentum, may be required for a structure to function in the role of a prefrontal cortex.

In order to demonstrate convincingly that brain structures in two different species are truly homologous, one must show that the resemblances between the two structures are not merely superficial and, further, one must establish the likelihood that a similar structure was present in a common ancestor ${ }^{16,17}$. In the case of PDL and prefrontal cortex, the presence of dopaminergic fibers in PDL provides only one point of resemblance to prefrontal cortex. It is necessary to show other resemblances in terms of connections, histochemistry and, ideally, embryonic development. For example, do PDL and prefrontal cortex have similar developmental 
histories, as do avian and mammalian basal ganglia ${ }^{18}$ ? Do birds have a dorsomedial thalamic nucleus and does it project to PDL? Since a hallmark of the mammalian dorsomedial thalamic nucleus is its prominent input from the ventral pallidum ${ }^{19}$, a comparable thalamic nucleus in birds should be evident as the projection target of the avian ventral pallidum, which was itself recently identified ${ }^{20}$. If birds do not have such a thalamic nucleus or have one that does not project to PDL, then the similarities between PDL and prefrontal cortex would begin to appear incidental. Does PDL project to the striatum of the basal ganglia, as does prefrontal cortex? The answer to this question (which was not discussed by Divac and Mogenson) is that a portion of PDL, termed the parieto-temporooccipital area (TPO), does ${ }^{21}$. However, this raises the possibility that not all of PDL is comparable to prefrontal cortex. Thus, more detailed anatomical studies and more comprehensive behavioral studies are required to determine both the extent of the region that shows similarity to mammalian prefrontal cortex and the degree of its similarity.

Further, to make the case that PDL and prefrontal cortex are homologous, it must be shown that a similar structure exists in modern reptiles, and hence may have existed in reptiles ancestral to birds and mammals. Previous studies in reptiles have not specifically looked for a prefrontal cortex, and so it is not known whether or not one is present. Finally, even if PDL and cortex are independently-evolved but functionally-similar structures, it will be important to demonstrate this by documenting more extensively the anatomical and functional similarities and by determining that the DA fiberrich regions of the hyperstriatum ventrale and archistriatum do not show greater anatomical and functional similarities to prefrontal cortex than does PDL.

Although the careful comparison of brain structures using multiple criteria can be laborious, such an approach has been successful in studying the evolution of several specific brain structures.
The basal ganglia of birds, reptiles and mammals have been shown to consist of the same basic neurotransmitter-specific and neuropeptide-specific neuronal populations, thereby suggesting that these structures have been present since the ancestral reptiles, if not earlier ${ }^{2}$. The implication of these findings is that the basal ganglia have performed similar functions since at least the evolutionary appearance of reptiles. The studies of R. G. Northcutt, T. H. Bullock, C. A. McCormick and D. A. Bodznick $^{22}$ on electroreception are another example of the value of the contemporary approach to evolutionary neuroscience. By carefully analysing the phylogenetic relationships among living electroreceptive fish and studying the details of the anatomical organization of the peripheral electroreceptive organs and their central targets, they found that the electroreceptive sense was an ancient characteristic of vertebrates, but one that was lost during the transition to land. They also found that the electroreceptive sense was lost independently by early ray-finned fish and reinvented in a few of the many orders of teleosts. Similarly, careful studies of PDL in birds, in conjunction with studies in reptiles, can clarify the evolutionary and functional relationship of PDL to prefrontal cortex. Regardless of the outcome of such studies, it remains clear that we have made great strides in clarifying the evolutionary relationships between the brains of mammals and non-mammals. In particular, studies in birds and reptiles have shown that the telencephala of birds, reptiles and mammals show many fundamental similarities. This climate has fostered the proposal of Divac and Mogenson, a proposal that would have been given scant consideration by the comparative neuroanatomists of the earlier part of this century but one that should receive more serious attention from contemporary neuroscientists.

\section{Acknowledgements}

I thank W. Hodos and S. E. Brauth for helpful discussions.

\section{Selected references}

1 Ariens-Kappers, C. U., Huber, G. C. and Crosby, E. C. (1936) The Comparative Anatomy of the Nervous System, Including Man, MacMillan

2 Reiner, A., Brauth, S. E and Karten, H. J. (1984) Trends Neurosci. 7, 320-325

3 Karten, H. J. (1969) Ann. N.Y. Acad. Sci. 16, 164-168

4 Nauta, W. J. H. and Karten, H. J. (1970) in The Neurosciences: Second Study Program (Schmitt, F. O., ed.), pp. 7-26, Rockefeller University Press

5 Parent, A. and Olivier, A. (1970) J. Hirnforsch. 12, 73-81

6 Karten, H. J. (1979) in Neural Mechanisms of Behavior in the Pigeon (Granda, A. M. and Maxwell, J. H., eds), pp. 409-430, Plenum Press

7 Hodos, W. (1976) Prog. Psychobiol. Physiol. Psychol. 6, 29-62

8 Benowitz, L. (1980) in Comparative Neurology of the Telencephalon (Ebbesson, S. O. E., ed.), pp. 389-421, Plenum Press

9 Ulinski, P. S. (1983) Dorsal Ventricular Ridge: A Treatise on Forebrain Organization in Reptiles and Birds, J. Wiley and Sons

10 Divac, I. and Mogenson, J. (1985) Neuroscience $15,677-682$

11 Mogenson, J. and Divac, I. (1982) Brain Behav. Evol. 21, 60-66

12 Divac, I., Bjorklund, A., Lindvall, $O$. and Passingham, R. E. (1978) J. Comp. Neurol. $180,59-72$

13 Wikmark, R. G. E., Divac, I. and Weiss, R. (1973) Brain Behav. Evol. 8, 329-339

14 Kolb, B. (1984) Brain Res. Rev. 8, 65-98

$15 \mathrm{Kitt}$, C. A. and Brauth, S. E. (1986) J. Comp. Neurol. 24, 91-110

16 Northcutt, R. G. (1984) Am. Zool. 24, 701-716

17 Campbell, C. B. G. and Hodos, W. (1970) Brain Behav. Evol. 3, 353-367

18 Kallen, B. (1962) Anat. Entw. Gesch. 36, $62-82$

19 Graybiel, A. M. (1984) in Functions of the Basal Ganglia (Everd, D. and O'Connor, M., eds), pp. 114-149, The Ciba Foundation and John Wiley \& Sons

20 Kitt, C. A. and Brauth, S. E. (1981) Neuroscience 6, 1551-1566

21 Brauth, S. E., Ferguson, J. L. and Kitt, C. A. (1978) Brain. Res. 147, 205-221

22 Bullock, T. H., Northcutt, R. G. and Bodznick, D. A. (1982) Trends Neurosci. $5,1 \cdots 4$

ANTON REINER

Department of Anatomy and Cell Biology, The University of Michigan, Ann Arbor, MI 48109. USA. 